\title{
Insensitivity to time-reversal symmetry breaking of universal conductance fluctuations with Andreev reflection
}

\author{
P. W. Brouwer and C. W. J. Beenakker \\ Instituut-Lorentz, University of Leiden, P.O. Box 9506, 2300 RA Leiden, The Netherlands
}

(Received 25 August 1995)

\begin{abstract}
Numerical simulations of conduction through a disordered microbridge between a normal metal and a superconductor have revealed an anomalous insensitivity of the conductance fluctuations to a magnetic field. A theory for the anomaly is presented: both an exact analytical calculation (using random-matrix theory) and a qualitative symmetry argument (involving the exchange of time-reversal for reflection symmetry).
\end{abstract}

Universal conductance fluctuations (UCF's) are a fundamental manifestation of phase-coherent transport in disordered metals. ${ }^{1,2}$ The adjective "universal" describes two aspects of the sample-to-sample fluctuations of the conductance: (1) The variance var $G$ is of order $\left(e^{2} / h\right)^{2}$, independent of sample size or disorder strength; and (2) var $G$ decreases precisely by a factor of 2 if time-reversal symmetry $(\mathcal{T})$ is broken by a magnetic field. The universality of this factor of 2 has been established both by diagrammatic perturbation theory ${ }^{1,2}$ and by randommatrix theory. ${ }^{3-6}$ In the former approach, one has two classes of diagrams, cooperons and diffusons, which contribute equally to var $G$ in the presence of $\mathcal{T}$. A magnetic field suppresses the cooperons but leaves the diffusons unaffected, hence var $G$ is reduced by $\frac{1}{2}$. In the latter approach, the universality of the factor-of- 2 reduction follows from the Dyson-Mehta theorem, ${ }^{7}$ which applies to the variance var $A$ of any observable $A=\sum_{n} a\left(T_{n}\right)$ that is a linear statistic on the transmission eigenvalues $T_{n} \cdot{ }^{8}$ The crossover from a linear to a quadratic eigenvalue repulsion upon breaking $\mathcal{T}$ directly leads to a reduction by $\frac{1}{2}$ of $\operatorname{var} A .^{9}$

The situation is qualitatively different if the normalmetal $(\mathrm{N})$ conductor is attached at one end to a superconductor (S). At the NS interface the dissipative normal current is converted into a dissipationless supercurrent via the scattering process of Andreev reflection: ${ }^{10}$ An electron incident from the normal-metal conductor is reflected as a hole, with the addition of a Cooper pair to the superconducting condensate. The conversion from normal to supercurrent has essentially no effect on the average conductance, provided that the interface resistance is negligibly small. ${ }^{11}$ However, the effect on the conductance fluctuations is striking: The variance is still universally of order $\left(e^{2} / h\right)^{2}$, but it has become insensitive to the breaking of $\mathcal{T}$. Numerical simulations by Marmorkos, Beenakker, and Jalabert ${ }^{12}$ of a disordered wire attached to a superconductor have shown that the variance is unaffected by a $\mathcal{T}$-breaking magnetic field, within the $10 \%$ statistical uncertainty of the simulations. This does not contradict the Dyson-Mehta theorem, because the conductance $G_{\mathrm{NS}}$ of the NS junction is a linear statistic in the presence - but not in the absence-of $\mathcal{T}^{13}$ One wonders whether there is some hidden symmetry principle that would constrain var $G_{\mathrm{NS}}$ to be the same, regardless of whether $\mathcal{T}$ is broken or not. No such symmetry principle has been found, and in fact we do not know of any successful generalization so far of the theory of UCF to quantities that are not linear statistics. ${ }^{14}$

Here we wish to announce that we have succeeded in the analytical calculation of var $G_{\mathrm{NS}}$ in the absence of $\mathcal{T}$, using techniques from random-matrix theory. We find that var $G_{\mathrm{NS}}$ for a disordered wire attached to a superconductor is reduced by $\left(2-90 / \pi^{4}\right)^{-1} \approx 0.929$ upon breaking $\mathcal{T}$. This number is sufficiently close to 1 to be consistent with the numerical simulations, ${ }^{12}$ and sufficiently different from 1 to explain why attempts to find a rigorous symmetry principle had failed. Still, we have been able to find an approximate symmetry argument that explains in an intuitively appealing way why the number $\left(2-90 / \pi^{4}\right)^{-1}$ is close to 1 . Our theory is more generally applicable than to a disordered wire: It applies to any NS junction for which the probability distribution $P(S)$ of the scattering matrix $S$ of the normal region depends only on the transmission eigenvalues $T_{n}$. (Such a distribution is called "isotropic." 6 ) As two examples, we consider a disordered metal grain and a ballistic constriction in a disordered wire. Our method can also be used to compute the effect of a magnetic field on weak localization in an NS junction, as reported elsewhere. ${ }^{15}$

The starting point of our calculation is the general relation between the conductance of the NS junction and the scattering matrix $S$ of the normal region, ${ }^{13}$

$$
\begin{aligned}
& G_{\mathrm{NS}}=2 G_{0} \operatorname{tr} m m^{\dagger}, \quad G_{0} \equiv 2 e^{2} / h, \\
& m=\sqrt{T}\left(1+u \sqrt{R} u^{*} \sqrt{R}\right)^{-1} u \sqrt{T}, \quad u \equiv w_{2} w_{1}^{*} .
\end{aligned}
$$

We used the polar decomposition

$$
S=\left(\begin{array}{ll}
v_{1} & 0 \\
0 & w_{1}
\end{array}\right)\left(\begin{array}{ll}
i \sqrt{R} & \sqrt{T} \\
\sqrt{T} & i \sqrt{R}
\end{array}\right)\left(\begin{array}{ll}
v_{2} & 0 \\
0 & w_{2}
\end{array}\right)
$$

where $v_{1}, v_{2}, w_{1}$, and $w_{2}$ are $N \times N$ unitary matrices $(N$ being the number of propagating modes at the Fermi level in each of the two leads attached to the sample). The matrix $T$ is a diagonal matrix with the $N$ transmission eigenvalues $T_{i} \in[0,1]$ on the diagonal, and $R$ is $1-T$. In the presence of $\mathcal{T}$, one has $S=S^{T}$, hence $w_{2}=w_{1}^{T}$, hence $u=1$. (The superscript $T$ denotes the transpose of the matrix.) Equation (1) then simplifies to ${ }^{13}$ 


$$
G_{\mathrm{NS}}(\mathcal{T})=2 G_{0} \sum_{n} T_{n}^{2}\left(2-T_{n}\right)^{-2},
$$

and $\operatorname{var} G_{\mathrm{NS}}$ follows directly from general formulas for the variance of a linear statistic on the transmission eigenvalues. ${ }^{8,16}$ In the absence of $\mathcal{T}$ no such simplification occurs.

To compute $\operatorname{var} G_{\mathrm{NS}}=\left\langle G_{\mathrm{NS}}^{2}\right\rangle-\left\langle G_{\mathrm{NS}}\right\rangle^{2}$ in the absence of $\mathcal{T}$, we assume an isotropic distribution ${ }^{17}$ of $S$, which implies that the average $\langle\cdots\rangle$ over the ensemble of scattering matrices can be performed in two steps: $\langle\cdots\rangle=\left\langle\langle\cdots\rangle_{u}\right\rangle_{T}$, where $\langle\cdots\rangle_{u}$ and $\langle\cdots\rangle_{T}$ are, respectively, the average over the unitary matrices $u$ and over the transmission eigenvalues $T_{2}$. It is convenient to add and subtract $\left\langle\left\langle G_{\mathrm{NS}}\right\rangle_{u}^{2}\right\rangle_{T}$, so that the variance of the conductance splits up into two parts,

$$
\begin{aligned}
\operatorname{var} G_{\mathrm{NS}}= & \left\langle\left\langle G_{\mathrm{NS}}\right\rangle_{u}^{2}\right\rangle_{T}-\left\langle\left\langle G_{\mathrm{NS}}\right\rangle_{u}\right\rangle_{T}^{2} \\
& +\left\langle\left\langle G_{\mathrm{NS}}^{2}\right\rangle_{u}-\left\langle G_{\mathrm{NS}}\right\rangle_{u}^{2}\right\rangle_{T},
\end{aligned}
$$

which we evaluate separately.

The first part is the variance of $\left\langle G_{\mathrm{NS}}\right\rangle_{u}$ over the distribution of transmission eigenvalues. As a consequence of the isotropy assumption, the matrix $u$ is uniformly distributed in the group $\mathcal{U}(N)$ of $N \times N$ unitary matrices. ${ }^{6}$ To evaluate $\left\langle G_{\mathrm{NS}}\right\rangle_{u}$ we need to perform an integral over $\mathcal{U}(N)$ of a rational function of $u$, according to Eq. (1). Such matrix integrals are notoriously difficult to evaluate in closed form, ${ }^{18}$ but fortunately we only need the large$N$ limit. Creutz ${ }^{19}$ and Mello ${ }^{20}$ have given general rules for the integral over $\mathcal{U}(N)$ of polynomial functions of $u$. By applying these rules we find that

$$
\begin{gathered}
\left\langle\operatorname{tr} T\left(u \sqrt{R} u^{*} \sqrt{R}\right)^{p} u T u^{\dagger}\left(\sqrt{R} u^{\mathrm{T}} \sqrt{R} u^{\dagger}\right)^{q}\right\rangle_{u} \\
=\delta_{p q} N \tau_{1}^{2}\left(1-\tau_{1}\right)^{2 p}+O(1)
\end{gathered}
$$

where we have defined the trace $\tau_{k}=N^{-1} \sum_{i} T_{i}^{k}$. It follows that, up to corrections of order unity,

$$
\left\langle G_{\mathrm{NS}}\right\rangle_{u}=2 G_{0} N \sum_{p=0}^{\infty} \tau_{1}^{2}\left(1-\tau_{1}\right)^{2 p}=\frac{2 G_{0} N \tau_{1}}{2-\tau_{1}} .
$$

Since $\tau_{k}$ is a linear statistic, we know that its fluctuations are an order $1 / N$ smaller than the average. ${ }^{6}$ This implies that, to leading order in $1 / N, \operatorname{var} f\left(\tau_{k}\right)=\left[f^{\prime}\left(\tau_{k}\right)\right]^{2} \operatorname{var} \tau_{k}$. The variance of Eq. (6) is therefore

$$
\left\langle\left\langle G_{\mathrm{NS}}\right\rangle_{u}^{2}\right\rangle_{T}-\left\langle\left\langle G_{\mathrm{NS}}\right\rangle_{u}\right\rangle_{T}^{2}=\frac{16 G_{0}^{2} N^{2} \operatorname{var} \tau_{1}}{\left(2-\left\langle\tau_{1}\right\rangle\right)^{4}}+O(1 / N)
$$

Note that the leading term in Eq. (7) is $O(1)$.

We now turn to the second part of Eq. (4), which involves the variance $\left\langle G_{\mathrm{NS}}^{2}\right\rangle_{u}-\left\langle G_{\mathrm{NS}}\right\rangle_{u}^{2}$ of $G_{\mathrm{NS}}$ over $\mathcal{U}(N)$ at fixed transmission eigenvalues and subsequently an average over the $T_{i}$ 's. The calculation is similar in principle to that described in the preceding paragraph, but many more terms contribute to leading order in $1 / N$. Here we only give the result

$$
\begin{aligned}
\left\langle\left\langle G_{\mathrm{NS}}^{2}\right\rangle_{u}-\left\langle G_{\mathrm{NS}}\right\rangle_{u}^{2}\right\rangle_{T}= & 16 G_{0}^{2}\left(2-\left\langle\tau_{1}\right\rangle\right)^{-6}\left\langle\tau_{1}\right\rangle^{-2}\left\{4\left\langle\tau_{1}\right\rangle^{2}-8\left\langle\tau_{1}\right\rangle^{3}+9\left\langle\tau_{1}\right\rangle^{4}-4\left\langle\tau_{1}\right\rangle^{5}+2\left\langle\tau_{1}\right\rangle^{6}\right. \\
& -4\left\langle\tau_{1}\right\rangle\left\langle\tau_{2}\right\rangle+2\left\langle\tau_{1}\right\rangle^{2}\left\langle\tau_{2}\right\rangle-2\left\langle\tau_{1}\right\rangle^{3}\left\langle\tau_{2}\right\rangle-2\left\langle\tau_{1}\right\rangle^{4}\left\langle\tau_{2}\right\rangle+6\left\langle\tau_{2}\right\rangle^{2}-6\left\langle\tau_{1}\right\rangle\left\langle\tau_{2}\right\rangle^{2} \\
& \left.+3\left\langle\tau_{1}\right\rangle^{2}\left\langle\tau_{2}\right\rangle^{2}-4\left\langle\tau_{1}\right\rangle\left\langle\tau_{3}\right\rangle+6\left\langle\tau_{1}\right\rangle^{2}\left\langle\tau_{3}\right\rangle-2\left\langle\tau_{1}\right\rangle^{3}\left\langle\tau_{3}\right\rangle\right\}+O(1 / N) .
\end{aligned}
$$

The sum of Eqs. (7) and (8) equals var $G_{\mathrm{NS}}$, according to Eq. (4). The resulting expression contains only moments of the transmission eigenvalues. This solves the problem of the computation of $\operatorname{var} G_{\mathrm{NS}}$ in the absence of $\mathcal{T}$, since these moments are known.

For the application to a disordered wire (length $L$, mean free path $\ell$ ) one has the variance ${ }^{2,5} N^{2} \operatorname{var} \tau_{1}=\frac{1}{15}$, and averages ${ }^{21}\left\langle\tau_{k}\right\rangle=\frac{1}{2}(\ell / L) \Gamma\left(\frac{1}{2}\right) \Gamma(k) / \Gamma\left(k+\frac{1}{2}\right)$. Substitution into Eqs. (7) and (8) yields (in the diffusive limit $\ell / L \rightarrow 0)$

$$
\operatorname{var} G_{\mathrm{NS}}(\text { no } \mathcal{T})=\frac{8}{15} G_{0}^{2} \approx 0.533 G_{0}^{2} .
$$

This is to be compared with the known result ${ }^{16}$ in the presence of $\mathcal{T}$,

$$
\operatorname{var} G_{\mathrm{NS}}(\mathcal{T})=\left(\frac{16}{15}-48 \pi^{-4}\right) G_{0}^{2} \approx 0.574 G_{0}^{2} .
$$

Breaking $\mathcal{T}$ reduces the variance by less than $10 \%$, as advertised.

We would like to obtain a more direct understanding of why the two numbers in Eqs. (9) and (11) are so close. To that end we return to the general expression (1) for the conductance $G_{\mathrm{NS}}$ of a NS junction, in terms of the scat- tering matrix $S$ of the normal region. We compare $G_{\mathrm{NS}}$ with the conductance $G_{\mathrm{NN}}$ of an entirely normal metal consisting of two segments in series (see Fig. 1). The first segment has scattering matrix $S$, the second segment is the mirror image of the first. That is to say, the disorder potential is specularly reflected and the sign of the magnetic field is reversed. The system NN thus has a reflection symmetry $(\mathcal{S})$, both in the presence and absence of $\mathcal{T}$. The scattering matrix of the second segment is $\Sigma S \Sigma$, where $\Sigma$ is a $2 N \times 2 N$ matrix with zero elements, except for $\Sigma_{i, N+i}=\Sigma_{N+i, i}=1(i=1,2, \ldots, N)$. (The matrix $\Sigma$ interchanges scattering states incident from left and right.) The conductance $G_{\mathrm{NN}}$ follows from the transmission matrix through the two segments in series by means of the Landauer formula,

$$
\begin{aligned}
& G_{\mathrm{NN}}(\mathcal{S})=G_{0} \operatorname{tr} m^{\prime} m^{\prime \dagger}, \\
& m^{\prime}=\sqrt{T}\left(1+u^{\prime} \sqrt{R} u^{\prime} \sqrt{R}\right)^{-1} u^{\prime} \sqrt{T}, \quad u^{\prime} \equiv w_{2} w_{1} .
\end{aligned}
$$

The difference between Eqs. (1) and (11) is crucial in the presence of $\mathcal{T}$, when $w_{2}=w_{1}^{T}$, so that $u=1$ while $u^{\prime}$ is some random (symmetric) unitary matrix. However, in the absence of $\mathcal{T}, w_{1}$ and $w_{2}$ are independent, so that 


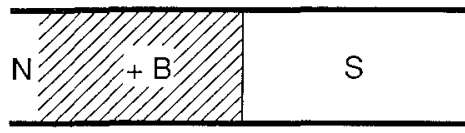

(a)

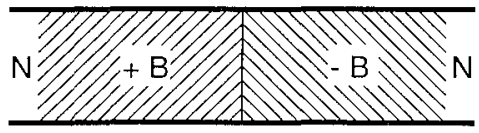

(b)

FIG. 1. (a) Schematic drawing of a disordered normal metal (N) connected to a superconductor (S), in a time-reversal symmetry $(\mathcal{T})$ breaking magnetic field $B$. In (b) the normal region is connected in series with its mirror image. As indicated, the magnetic field $B$ changes sign upon reflection. The variance of the conductance fluctuations in (a) is exactly four times the variance in (b). The variance in (b) is exactly two times the variance in the absence of the reflection symmetry $(\mathcal{S})$. The exchange of $\mathcal{T}$ for $\mathcal{S}$ explains the insensitivity of the conductance fluctuations to a magnetic field, as discussed in the text.

both $u$ and $u^{\prime}$ are randomly distributed unitary matrices. We have repeated the calculation of the variance starting from Eq. (11), and found that var $\operatorname{tr} m m^{\dagger}=\operatorname{var} \operatorname{tr} m^{\prime} m^{\prime \dagger}$, hence

$$
\operatorname{var} G_{\mathrm{NS}}(\text { no } \mathcal{T})=4 \operatorname{var} G_{\mathrm{NN}}(\mathcal{S}, \text { no } \mathcal{T}) .
$$

The system NN is special because it possesses a reflection symmetry. Breaking $\mathcal{S}$ amounts to the replacement of the mirror-imaged segment by a different segment, with scattering matrix $S^{\prime}$ which is independent of $S$ but drawn from the same ensemble. Breaking $\mathcal{S}$ reduces the variance of the conductance fluctuations by a factor of 2 , regardless of whether $\mathcal{T}$ is present or not,

$$
\operatorname{var} G_{\mathrm{NN}}(\mathcal{S})=2 \operatorname{var} G_{\mathrm{NN}}(\text { no } \mathcal{S}) .
$$

We have checked this relation by an explicit calculation, but it seems intuitively obvious if one considers that the eigenstates separate into even and odd states that fluctuate independently. Since breaking $\mathcal{T}$ by itself reduces the variance of $G_{\mathrm{NN}}$ by a factor of 2 , we may write

$$
\operatorname{var} G_{\mathrm{NN}}(\mathcal{S}, \text { no } \mathcal{T})=\operatorname{var} G_{\mathrm{NN}}(\mathcal{T}, \text { no } \mathcal{S}) .
$$

Equations (12)-(14) are exact, and hold for any isotropic distribution of the scattering matrix. We need one more relationship, which is approximate and holds only for the case of a disordered wire: ${ }^{13,22}$

$$
\operatorname{var} G_{\mathrm{NS}}(\mathcal{T}) \approx 4 \operatorname{var} G_{\mathrm{NN}}(\mathcal{T}, \text { no } \mathcal{S}) .
$$

Taken together, Eqs. (12)-(15) imply the approximate relationship $\operatorname{var} G_{\mathrm{NS}}(\mathcal{T}) \approx \operatorname{var} G_{\mathrm{NS}}($ no $\mathcal{T})$. The exact calculation shows that the approximation is accurate within $10 \%$. We now understand the insensitivity of the conductance fluctuations of a (disordered) NS junction to a magnetic field as an exchange of symmetries in the related normal system NN: As $\mathcal{T}$ is broken, $\mathcal{S}$ is established, thereby compensating the reduction of $\operatorname{var} G_{\mathrm{NS}} \cdot{ }^{23}$

We have emphasized the general applicability of Eqs. (7), (8), and (12)-(14), which hold not just for a disordered wire, but for any isotropic distribution of the

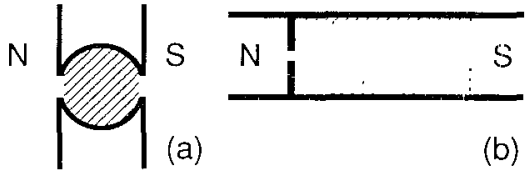

FIG. 2. (a) Schematic drawing of an NS junction consisting of a disordered metal grain (shaded). (b) A disordered normal-metal wire (shaded) containing a point contact.

scattering matrix. We illustrate this by two examples. The first is an NS junction consisting of a disordered metal grain [see Fig. 2(a)]. The coupling of a normal metal and a superconductor to the grain occurs via ballistic point contacts (width much smaller than the mean free path in the grain). Following Ref. 24, we may assume that the scattering matrix of the grain is distributed according to the circular ensemble of random-matrix theory. This is an isotropic distribution. The relevant moments of the transmission eigenvalues in the absence of $\mathcal{T} \operatorname{are}^{24}\left\langle\tau_{k}\right\rangle=\pi^{-1 / 2} \Gamma\left(k+\frac{1}{2}\right) / \Gamma(k+1), N^{2} \operatorname{var} \tau_{1}=\frac{1}{16}$. Substitution into the general formulas (7) and (8) yields

$$
\operatorname{var} G_{\mathrm{NS}}(\text { no } \mathcal{T})=\frac{128}{243} G_{0}^{2} \approx 0.527 G_{0}^{2},
$$

which is again close to the known result in the presence of $\mathcal{T},{ }^{24}$

$$
\operatorname{var} G_{\mathrm{NS}}(\mathcal{T})=\frac{9}{16} G_{0}^{2} \approx 0.563 G_{0}^{2}
$$

The second example is a ballistic constriction (point contact) in a wire that is connected to a superconductor [see Fig. 2(b)]. The point contact has conductance $N_{0} G_{0}$, which we assume to be much smaller than the conductance $N \ell / L$ of the disordered wire by itself. As discussed in Ref. 25, we may assume an isotropic distribution of the scattering matrix of the combined system (point contact plus disordered wire). The moments of the transmission eigenvalues $\operatorname{are}^{25}\left\langle\tau_{k}\right\rangle=N_{0} / N, N^{2} \operatorname{var} \tau_{1}=$ $O\left(N_{0} L / N \ell\right)^{2}$. Substitution into Eqs. (7) and (8) yields, in the limit $N_{0} L / N \ell \rightarrow \mathbf{0}$,

$$
\operatorname{var} G_{\mathrm{NS}}(\text { no } \mathcal{T})=\frac{1}{2} G_{0}^{2} .
$$

In contrast, if $\mathcal{T}$ is not broken, the conductance fluctuations are suppressed in this limit: ${ }^{25,26}$

$$
\operatorname{var} G_{\mathrm{NS}}(\mathcal{T})=O\left(N_{0} L / N \ell\right)^{2} \ll G_{0}^{2} .
$$

In this geometry a magnetic field greatly enhances the conductance fluctuations. The reason that a disordered wire with a constriction behaves so differently from an unconstricted wire is that the relation (15) does not hold in the presence of a constriction. However, the general relationship (12) does hold, and indeed the result (18) is four times the variance of a structure consisting of two point contacts in series with a reflection symmetry.

In summary, we have solved the problem of universal conductance fluctuations in normal-metalsuperconductor junctions in a magnetic field, under the assumption of an isotropic distribution of the scattering matrix of the normal region. We find that the 
structure of the scattering matrix of the normal-metalsuperconductor junction in the absence of time-reversal symmetry allows one to relate the conductance fluctuations to those of a normal system with reflection symmetry. This reflection symmetry is absent in the presence of time-reversal symmetry. It compensates the reduction of the conductance fluctuations due to breaking of time- reversal symmetry, and explains the anomalous insensitivity of the fluctuations in a magnetic field discovered in computer simulations. ${ }^{12}$

Discussions on this problem with A. Altland are gratefully acknowledged. This work was supported by the Dutch Science Foundation NWO/FOM.
${ }^{1}$ B. L. Al'tshuler, Pis'ma Zh. Eksp. Teor. Fiz. 41, 530 (1985) [JETP Lett. 41, 648 (1985)].

${ }^{2}$ P. A. Lee and A. D. Stone, Phys. Rev. Lett. 55, 1622 (1985).

${ }^{3}$ Y. Imry, Europhys. Lett. 1, 249 (1986).

${ }^{4}$ K. A. Muttalib, J.-L. Pichard, and A. D. Stone, Phys. Rev. Lett. 59, 2475 (1987).

${ }^{5}$ P. A. Mello, Phys. Rev. Lett. 60, 1089 (1988).

${ }^{6}$ A. D. Stone, P. A. Mello, K. A. Muttalib, and J.-L. Pichard, in Mesoscopic Phenomena in Solids, edited by B. L. Al'tshuler, P. A. Lee, and R. A. Webb (North-Holland, Amsterdam, 1991).

${ }^{7}$ F. J. Dyson and M. L. Mehta, J. Math. Phys. 4, 701 (1963).

${ }^{8}$ C. W. J. Beenakker, Phys. Rev. Lett. 70, 1155 (1993).

${ }^{9}$ Here, and in the rest of the paper, we assume that there is no spin-orbit interaction, and that spin-rotation symmetry is maintained both with and without $\mathcal{T}$.

${ }^{10}$ A. F. Andreev, Zh. Eksp. Teor. Fiz. 46, 1823 (1964) [Sov. Phys. JETP 19, 1228 (1964)].

${ }^{11}$ For a review, see C. W. J. Beenakker, in Mesoscopic Quantum Physics, edited by E. Akkermans, G. Montambaux, J.-L. Pichard, and J. Zinn-Justin (North-Holland, Amsterdam, 1995).

${ }^{12}$ I. K. Marmorkos, C. W. J. Beenakker, and R. A. Jalabert, Phys. Rev. B 48, 2811 (1993).

${ }^{13}$ C. W. J. Beenakker, Phys. Rev. B 46, 12841 (1992).

${ }^{14} \mathrm{~A}$ promising field-theoretic approach to this problem, based on the mapping onto a supersymmetric nonlinear $\sigma$ model, has so far not been successful [A. Altland (private communication)]. The more conventional diagrammatic perturbation theory suffers from a proliferation of relevant diagrams, and has so far not been completed even in the presence of $\mathcal{T}$ [Y. Takane and H. Ebisawa, J. Phys. Soc. Jpn. 60, 3130 (1991)].

${ }^{15}$ P. W. Brouwer and C. W. J. Beenakker, Phys. Rev. B 52, 3868 (1995).
${ }^{16}$ C. W. J. Beenakker and B. Rejaei, Phys. Rev. Lett. 71, 3689 (1993); J. T. Chalker and A. M. S. Macêdo, ibid. 71, 3693 (1993).

${ }^{17}$ The assumption of an isotropic distribution of $S$ is sufficient but not necessary. A weaker assumption that also ensures that the matrix $u$ in Eq. (1b) is uniformly distributed in $\mathcal{U}(N)$ is the so-called "equivalent channel assumption" [P. A. Mello and S. Tomsovic, Phys. Rev. Lett. 67, 342 (1991)]. Microscopic models that satisfy this assumption have been given by O. N. Dorokhov [Phys. Rev. B 37, 10526 (1988)] and S. Iida, H. A. Weidenmüller, and J. A. Zuk [Ann. Phys. (N.Y.) 200, 219 (1990)].

${ }^{18}$ L. K. Hua, Harmonic Analysis of Functions of Several Complex Variables in the Classical Domains (American Mathematical Society, Providence, RI, 1963).

${ }^{19}$ M. Creutz, J. Math. Phys. 19, 2043 (1978).

${ }^{20}$ P. A. Mello, J. Phys. A 23, 4061 (1990).

${ }^{21}$ C. W. J. Beenakker and M. Büttiker, Phys. Rev. B 46, 1889 (1992).

${ }^{22}$ Y. Takane and H. Ebisawa, J. Phys. Soc. Jpn. 61, 2858 (1992).

${ }^{23}$ To avoid misunderstandings concerning the "exchange of symmetries," we stress that it refers to the related NN system and not to the NS junction itself. To be precise, the structure of the scattering matrix of the NS junction is such that the NS junction in the absence of $\mathcal{T}$ is related to an NN system with $\mathcal{S}$, whereas in the presence of $\mathcal{T}$ it is related to an NN system without $\mathcal{S}$. The first relationship is exact [Eq. (12)], the second one is approximate [Eq. (15)].

${ }^{24}$ H. U. Baranger and P. A. Mello, Phys. Rev. Lett. 73, 142 (1994); R. A. Jalabert, J.-L. Pichard, and C. W. J. Beenakker, Europhys. Lett. 27, 255 (1994).

${ }^{25}$ C. W. J. Beenakker and J. A. Melsen, Phys. Rev. B 50, 2450 (1994).

${ }^{26}$ D. L. Maslov, C. Barnes, and G. Kirczenow, Phys. Rev. Lett. 70, 1984 (1993). 